\title{
Impactos económicos da capacidade e regra operacional do sistema hídrico da parte moçambicana da bacia do rio limpopo para satisfação das actuais e futuras demandas de água na agricultura irrigada
}

\author{
Economic impacts of operational rules and capacity of the mozambican \\ part of limpopo river basin water system to meet current \\ and future water demands in irrigated agriculture
}

\section{Virgílio António Livele André Luiz Lopes da Silveira}

RESUMO: Este trabalho aplicou o modelo de simulação Acquanet, através do seu submodelo de Análise Económica, com objectivo de medir e avaliar os impactos económicos obtidos pelos usuários agrícolas na operação do sistema hídrico da parte moçambicana da bacia do Limpopo. A pesquisa visa auxiliar, dentro das condiçóes operacionais do sistema analisado, aos produtores agrícolas na percepção dos benefícios económicos ganhos ou que podem ser alcançados com a satisfação das suas necessidades hídricas, e dos custos associados em caso de escassez da água. Os resultados mostraram que o sistema hídrico em estudo consegue satisfazer a $100 \%$ todas demandas actuais dos usuários agrícolas, o que possibilita a estes obterem os possíveis benefícios económicos máximos anuais advindos da água alocada. Entretanto, o desempenho do mesmo sistema apresenta-se incapaz de poder acomodar as demandas projectadas para 2017 e satisfazer na sua totalidade todas necessidades de água, o que consequentemente poderá gerar perdas económicas devido ao fornecimento de água abaixo dos volumes demandados. Observou-se ainda das análises efectuadas que, dependendo da disponibilidade hídrica, a operação da barragem de Massingir na sua capacidade máxima (nível pleno de armazenamento), pode servir como solução alternativa para a minimização futura dos déficits de água no atendimento de todos os principais usuários naquela área de estudo.

PALAVRAS-CHAVE: operação do sistema hídrico; usuários agrícolas; benefícios económicos.
Submetido: 29-06-15

Revisão: 01-07-15

Aprovado: 03-07-15
ABSTRACT: This paper applied the economic analysis submodel of the Acquanet simulation model to measure and evaluate the economic impacts obtained by agricultural users in the operation of the Mozambican part of the Limpopo river basin water system. The research aims to assist farmers, within the operating conditions of the analyzed system to perceive the economic benefits or gains that can be achieved by meeting their water needs, and associated costs when there is water shortage. The results showed that the water system studied can meet $100 \%$ of all current demands of agricultural users, enabling them to achieve the possible maximum economic benefits from annual allocated water. However, the performance of the system is unable to accommodate the demands projected for 2017 and meet all water needs in full, which consequently could generate economic losses due to the water supply below the volumes demanded. It was also observed in the analyses carried out, that, depending on the availability of water, the operation of the Massingir dam at its maximum capacity (full storage level), can serve as an alternative solution to minimize future water deficits in serving all the main users in the area studied.

KEYWORDS: water system operation; agricultural users; economic benefits.

\section{INTRODUÇÃO}

A agricultura, o sector que mais água demanda no mundo, constitui a base de desenvolvimento e alavanca das economias de vários países, sobretudo os em via de desenvolvimento, dos quais, Moçambique (onde se localiza o objecto de estudo) faz parte. Assim, são necessárias políticas nacionais e técnicas 
científicas para o gerenciamento da disponibilidade e uso eficiente de água, de tal sorte que se permita uma intensificação sustentável desta actividade sócioeconómica.

Bogaert et al. (2012), ressalta a primordialidade da agricultura na sociedade moderna pela sua principal importância de proporcionar alimentos e garantir a segurança alimentar. Porém, esta mesma fonte, chama atenção especial para o sector de irrigação agrícola por ser o usuário que utiliza maiores quantidades de água quando comparado com os outros sectores de economia no mundo que tem a água como um dos principais factores de produção.

A Organização das Nações Unidas para Alimentação e Agricultura (FAO) corrobora com Bogaert et al. (2012), estimando um consumo na agricultura irrigada de cerca de $70 \%$ da água doce disponível. Este nível de consumo, associado a um futuro incerto quanto aos índices de crescimento populacional, pressão pela busca dos alimentos, ocorrência das mudanças climáticas (CALIFORNIA WATER PLAN, 2009), torna incontornável a disponibilidade restrita dos recursos hídricos e o aumento das demandas em todos sectores de actividades económicas.

Perante esta situação dicotômica, oferta já limitada de água, enfrentando o aumento dos níveis de abstracção deste recurso para diferentes sectores que tem a água como insumo de produção, principalmente na agricultura irrigada com maior consumo e a sua imprescindibilidade na vida do Homem, impóe-se a necessidade de se avaliar os possíveis impactos económicos na operação de sistemas hídricos para satisfação das demandas de água entre os usuários.

Em quase todo mundo, os decisores políticos, gestores dos recursos hídricos, e inclusive os economistas, através de estimativas e interpretação de funçôes de demanda ou benefícios marginais advindos do uso da água, procuram compreender o comportamento dos utilizadores de água, visando garantir um equilíbrio entre oferta e demanda deste recurso nos diferentes sectores de actividades.

Desde a ênfase colocada pelas conferências de Dublin em Janeiro de 1992, e do Rio, em Junho do mesmo ano, sobre o tratamento da água como um bem económico, Molle e Berkoff (2007), apontam que muita esperança tem sido investida na valoração económica da água através de sua precificação, como um meio de regular e racionalizar o uso dos recursos hídricos em diferentes sectores.
Para Young (1996), particularmente na agricultura irrigada, valor económico da água utilizada no processo produtivo é uma medida dos benefícios económicos de contribuição desta água para o valor da produçáo agrícola. No entanto, é com o conhecimento deste valor ou benefício económico da água alocada para os usuários, que estes podem ser induzidos a um uso mais racional e maximizarem a productividade em cada $\mathrm{m}^{3}$ disponibilizado.

Este trabalho, cujo principal objectivo foi de medir e avaliar os impactos económicos obtidos pelos agricultores na operação do sistema hídrico da parte moçambicana da bacia do rio Limpopo para a satisfação das necessidades de água nas actividades agrícolas, é justificado pela sua importância na conscientização dos usuários de água neste sector sobre o valor real dos recursos hídricos, o que pode, sobremaneira, motivar na mudança do comportamento dos agricultores no uso perdulário da água, induzindo-os a um uso de água mais racional e maximizaçáo da productividade em cada $\mathrm{m}^{3}$ alocado. Por outro lado, as entidades e/ ou aos gestores que fazem o planejamento e gestão da água e das infraestruturas dos sistemas hídricos poderão ter o valor económico como mais uma base de decisão na oferta e alocação de água entre os usuários.

Para a avaliação económica da água utilizada na área de estudo, foram determinadas curvas de demandas de água dos principais usuários agrícolas (económicos) através do método Point Expansion, e simulou-se diferentes cenários no modelo Acquanet para a determinaçáo dos benefícios ganhos pelos agricultores nas quantidades de água alocada e possíveis custos de escassez em caso de atendimento abaixo das vazóes de água demandas pelos usuários considerados.

\section{Método Point Expansion}

Segundo Griffin (2006), o método Point Expansion é uma técnica de fácil aplicação e capaz de fornecer uma estimativa de função de demanda em contextos de recursos hídricos. Para a utilização deste método deve ser conhecido um ponto na funçáo de demanda (w-quantidade da água, p-preço), informação geralmente disponível, e um valor estimado da elasticidade preço da demanda (ou sua inclinaçáo) através de um outro método. No entanto, o método apenas confere maior confiabilidade quando as estimativas são muito próximas do ponto real de expansão da curva de demanda, e quanto mais distante desse ponto a curva torna-se náo confiável e a sua aplicação também pouco fiável. 
Porque a elasticidade preço da demanda e o ponto de demanda ( $w, p)$, por si constituem apenas dois parâmetros, a estimativa da curva de demanda é de funçóes com dois parâmetros, especialmente as formas linear (1) e de elasticidade constante (potencial) (2):

$$
w=m^{*} p+b
$$

ou

$$
w=k^{*} p^{\varepsilon}
$$

Onde: $w$ - quantidade de água demandada; $p$ - custo da água; $m$ e $b$ coeficientes da função linear; $k$ - constante da função potencial e $\varepsilon$ - elasticidade-preço de demanda.

Destas duas expressões, a fórmula 2 pode também ser chamada de linear-log ou log-log, pois permite uma transformação logarítmica no seu lado direito e produz um relacionamento que é linear em p. E ambas funções podem ser facilmente invertidas para p, possibilitando a determinação dos benefícios totais obtidos pelo usuário. Baseando-se ainda em Griffin (2006), apresenta-se a seguir uma súmula desenvolvimento analítico do método.

A elasticidade-preço da demanda $(\varepsilon)$, de interesse neste trabalho, é definida como a variação percentual na quantidade demandada que irá ocorrer para uma variação percentual no preço, podendo ser determinada com a expressão:

$$
\varepsilon=\frac{\frac{\Delta w}{w}}{\frac{\Delta p}{p}}=\frac{\Delta w}{\Delta p} * \frac{p}{w}
$$

Porque $\mathbf{w}$ demandada cai com a subida do $\mathbf{p}, \varepsilon$ é negativa. E no limite, como a variaçáo percentual no preço torna-se pequeno, para que se possa falar de elasticidade-preço em um ponto específico na funçáo de demanda, pode se reescrever a $\varepsilon$ com a seguinte fórmula:

$$
\varepsilon=\frac{d w}{d p} * \frac{p}{w}
$$

É importante fazer menção que, geralmente as funções de demanda hídrica são preço inelásticas, o que significa que: $-1<\varepsilon<0$. E considera-se demanda elástica quando a elasticidade for menor que -1 .

Assumindo uma demanda linear e a elasticidade-preço em um ponto específico na função, a expressão 4 , onde $\frac{d w}{d p}$ representa a inclinação ou declividade (m) que caracteriza a reacção do usuário perante uma variação no preço da água, e conhecendo-se um ponto de demanda ( $w, p)$ e elasticidade estimada, podem ser determinados os parâmetros $\mathbf{m}$ e $\mathbf{b}$ e obter-se a função de demanda linear (1):

$$
\frac{d w}{d p}=m=\frac{\varepsilon}{\frac{p}{w}} \quad \text { e } \quad b=w-m^{*} p
$$

Esses dois parâmetros determinados, são incorporados na expressáo da curva linear e consegue-se assim a função: $w=m p+b$.

Como alternativa à função linear (1), tem-se a expressão potencial (2) que é obtida ao se assumir elasticidade-preço de demanda constante ao longo de todo domínio da função e, é operada a fórmula (4), primeiro em uma equação diferenciável, e depois

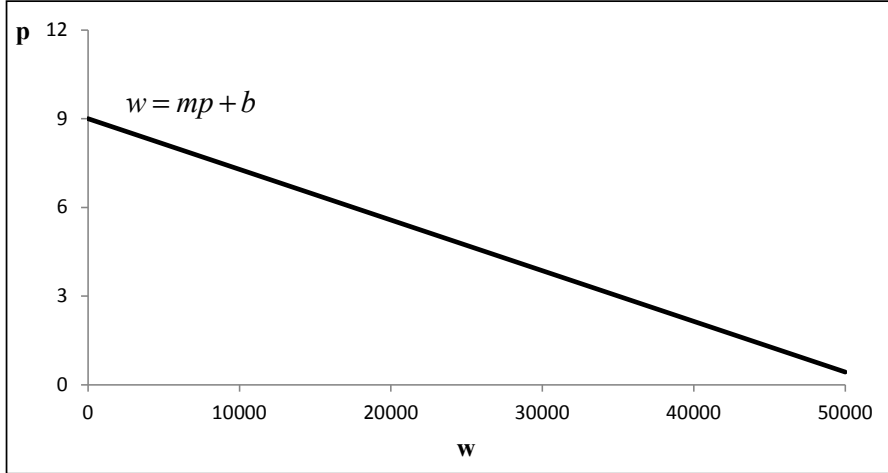

FIGURA 1. Curva da Função Produção linear determinada através do Point Expansion. 
resolvida por via da integração. Substituindo o ponto e o valor elasticidade em (2) é possível determinar o valor de k:

$$
\begin{gathered}
\frac{d w}{w}=\varepsilon^{*} \frac{d p}{p} \\
\int \frac{d w}{w}=\varepsilon^{*} \int \frac{d p}{p} \\
\ln w=\varepsilon^{*} \ln p+\ln k
\end{gathered}
$$

Onde ln $k$ é uma constante arbitrária, então obtém-se a função da curva demanda de elasticidade constante: $w=k p^{\varepsilon}$.

Com as duas funçóes de demanda supraditas, que também podem ser chamadas de curvas de benefício marginal ou disponibilidade a pagar pelo uso da água, é possível determinar os benefícios totais $(b m g)$ através de sua integração ao longo da curva. Para tal, ambas as funçôes devem antes ser invertidas e resolvidas em ordem a p:

$$
\begin{array}{rc}
w=m p+b & w=k p^{\varepsilon} \\
p=\frac{w-b}{m} & \text { ou } \\
p=b m g=\int_{w_{i}}^{w_{i+1}} \frac{w-b}{m} d w & (9) \quad p=b m g=\int_{w_{i}}^{\frac{1}{\varepsilon}}\left(\frac{w}{k}\right)^{\frac{1}{\varepsilon}} d w
\end{array}
$$

Importa realçar que a técnica de Point Expansion para estimativa da função de demanda da água, assim como outros métodos, oferece apenas uma estimativa da reacção do consumidor em relação a variaçáo do preço, sem no entanto conseguir descrever o comportamento real humano, pois este toma suas próprias decisôes, que provavelmente não seguirão rigorosamente nenhuma das duas funçóes.

\section{O Sistema de Suporte a Decisões (SSD) Acquanet}

O Acquanet é um modelo de rede de fluxo para simulação e análise de sistemas complexos em recursos hídricos, e auxiliar no processo de tomada de decisóes em problemas relacionados ao aproveitamento de água. Este sistema é uma versão brasileira desenvolvida pelo LabSid, da Escola Politécnica da USP (LABSID, 2002), a partir do modelo original MODSIM (LABADIE, 2010).

Como princípio básico de utilização, o SSD Acquanet deve ter a representação de sistemas de recursos hídricos em rede de fluxo, no qual os seus componentes são os nós (reservatórios, pontos de demandas, confluências e pontos de desvios) e os elos ou arcos de ligação (trechos naturais dos rios, canais e condutos) (ROBERTO, 2002).

Este SSD, pese embora ser um modelo de simulação, possui um algorítmo chamado Out-of-kilter que minimiza o custo de transporte de água na rede de fluxo, fazendo uma otimização para cada intervalo de tempo ( $\mathrm{t}$ $=1, \ldots ., \mathrm{T})$ considerado no sistema em análise (ROBERTO; PORTO 2001; ROBERTO et al., 2007). A seguir apresenta-se a descrição matemática do algorítmo" :

Função Objectivo (FO) a minimizar:

$$
\min \sum_{(i, j) \in A} c_{i j} x_{i j}
$$

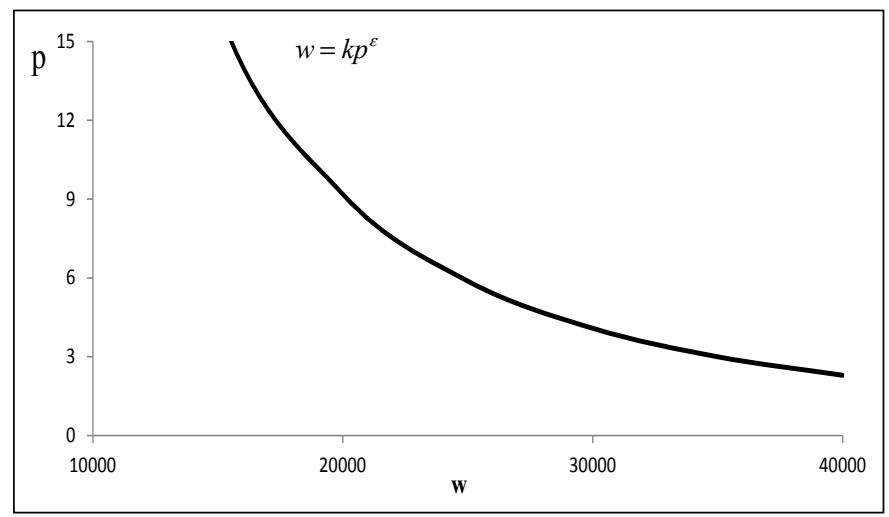

FIGURA 2. Curva de demanda na forma de Elasticidade Constante (Potencial) determinada através do Point Expansion. 
Sujeita às seguintes equações de restrição:

$$
\begin{aligned}
& \sum_{\frac{\{j \mid(i, j) \in A\}}{O U T}} x_{i j}-\sum_{\frac{\{j \mid(j, i) \in A\}}{I N} x_{j i}}=0 \quad i=1, \ldots ., N \\
& l_{i j} \leq x_{i j} \quad i, j=1, \ldots \ldots, N \\
& x_{i j} \leq u_{i j} \quad i, j=1, \ldots \ldots, N
\end{aligned}
$$

Onde:

$x_{i j}$ - representa a taxa de fluxo no arco $(\mathrm{i}, \mathrm{j})$ com os parâmetros do elo $\left[l_{i j} ; u_{i j} ; c_{i j}\right]$;

$c_{i j}$ - é o custo por unidade de fluxo no arco $(\mathrm{i}, \mathrm{j})$;

$l_{i j}, u_{i j}$ - são respectivamente, os limites inferiores e superiores do trecho do fluxo;

$A$ - é o conjunto de todas ligaçóes ou arcos na rede;

$\mathrm{N}$ - é o número total de nós.

A eq. 11 representa a FO que vai minimizar o custo de transporte do fluxo pelos arcos na rede;

¿ A primeira equação das condiçóes de restrição (12) garante o balanço de massa em todos os nós $i=1, \ldots . ., N$, isto é, a vazão de entrada deve ser igual a de saída, inclusive nos nós automaticamente criados;

I A eq. 13 indica $\mathrm{a}$ restrição do fluxo mínimo no trecho, podendo ser maior ou igual a capacidade inferior do arco;

A eq. 14 refere-se a restrição do fluxo máximo no trecho, devendo ser menor ou igual a capacidade superior do elo.

\section{FIGURA 3. Divisão da bacia hidrográfica do Limpopo entre os quatro países. Fonte: elaborado pelo autor; 2015.}

É ainda de capital relevância fazer menção que o Acquanet, tal como outros modelos desenvolvidos no LabSid (MODSIMP32), faz as simulaçôes de maneira sequencial no tempo (Simulação Contínua) ou estatisticamente (Planejamento Táctico), e apresenta duas opçóes de cálculo: Estados Hidrológicos e Calibração (LABSID, 2002; PORTO et al., 2003). E em relação à sua constituição, este modelo, para além do módulo base, apresenta mais cinco principais submodelos: (i) Alocação de Água; (ii) Qualidade da Água; (iii) Irrigação; (iv) Produção de Energia Hidroeléctrica e (v) Análise Económica na Alocação (PORTO et al., 2003).

\section{METERIAL E MÉTODOS}

\section{Descrição do objecto de estudo - "parte moçambicana da bacia do rio Limpopo"}

A bacia hidrográfica do rio Limpopo, com o segundo maior rio da África Austral, situa-se aproximadamente entre os paralelos $22^{\circ}$ e $26^{\circ}$ Sul e os meridianos $26^{\circ}$ e $35^{\circ}$ Este. A parte moçambicana, estudada neste trabalho, localiza-se entre os paralelos $21^{\circ}$ e $25^{\circ} \mathrm{Sul}$ e os meridianos de $31^{\circ}$ e $35^{\circ}$ Este (DNA,1996).

Esta bacia é compartilhada por quatro países (figura 3): África do Sul, Moçambique, Botswana e

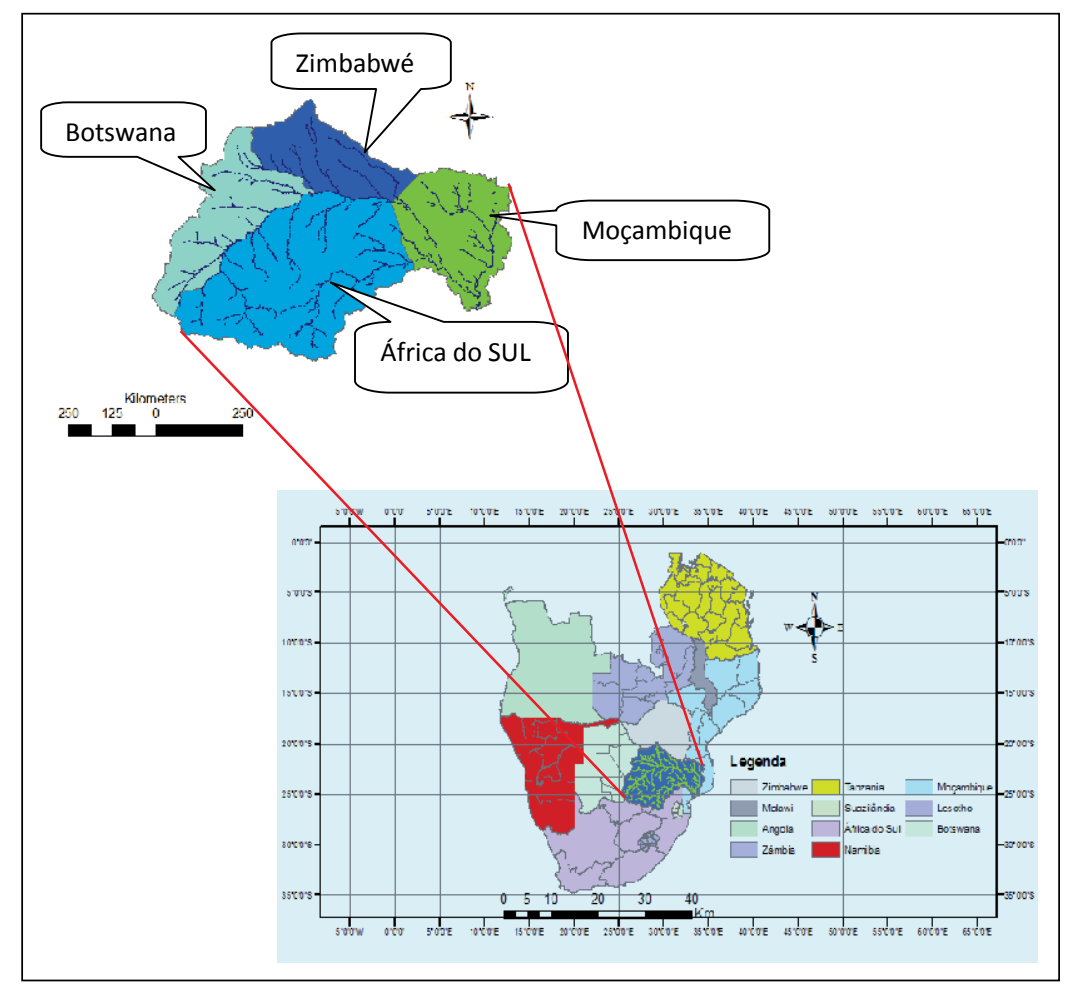


Zimbabwé. Ela nasce a Oeste da cidade de Pretória, na África do Sul, onde o seu rio é formado pela confluência dos rios Great Marico e Crocodilo, a cerca de $1500 \mathrm{~m}$ de latitude.

O rio Limpopo, com uma extensão de $1770 \mathrm{~km} \mathrm{e}$ uma área de $408250 \mathrm{Km}^{2}$ distribuída entre os quatro países (tabela 1), flui desde a província do Limpopo, na África do Sul, passa por Moçambique e escoa até o oceano Índico, LBPTC (2010).

TABELA 1

Distribuição das respectivas áreas entre os quatro países pertencentes a bacia do Limpopo

\begin{tabular}{|l|c|c|}
\hline \multicolumn{1}{|c|}{ País } & Área $\left.\mathbf{( k m}^{\mathbf{2}}\right)$ & $\%$ \\
\hline África do Sul & 184.150 & 45 \\
\hline Moçambique & 79.800 & 19,5 \\
\hline Botswana & 81.400 & 20 \\
\hline Zimbabwé & 62.900 & 15,5 \\
\hline Total & 408.250 & 100 \\
\hline
\end{tabular}

Fonte: (LBPTC, 2010).

A parte da bacia do Limpopo em Moçambique, com uma área de $79.800 \mathrm{~km}^{2}$, ocupa toda província de Gaza e uma pequena parte da província de Inhambane, englobando três grandes sub-bacias (figura 4). A sub-bacia do Changane que localiza-se inteiramente no território nacional. A sub-bacia do Elefantes com o rio que nasce nas zonas altas da África do Sul e a sub-bacia do Limpopo, que possui o curso principal do rio Limpopo dentro do território moçambicano.
Conforme Brito et al. (2006) a região da bacia do rio Limpopo é caracterizada pela predominância de um clima fortemente influenciado pela zona de altas pressóes localizada no oceano Índico à leste e, no oceano Atlântico à oeste, apresentando duas estaçôes distintas: a estação seca e fria, entre abril e setembro; e a quente e húmida, de outubro a março. A sua variação é de clima tropical seco de savana a quente seco de estepe (LBPTC, 2010). E na parte moçambicana da bacia, o clima varia de semi-árido húmido a árido, respectivamente da costa para o interior. A bacia, influenciado por este clima que se distingue na regiâo, apresenta uma evapotranspiração média que varia entre 1000 a $2000 \mathrm{~mm}$ por ano.

Com uma precipitaçáo média anual de $530 \mathrm{~mm}$ por ano, variando entre 200 a $1200 \mathrm{~mm} / \mathrm{a}$, as chuvas dentro da bacia registam-se mais na época húmida $\mathrm{e}$ quente, chegando a representar 76 a $84 \%$ do total da precipitaçáo anual, com o pico desta estação no mês de janeiro (BRITO et al., 2006). E a estação seca e fria, com cerca de 24 a $26 \%$ do total da precipitação anual. No território moçambicano tem-se uma média anual de $535 \mathrm{~mm}$, e a chuva varia entre $1000 \mathrm{~mm}$ na zona costeira e $350 \mathrm{~mm}$ em Pafuri, apresentando uma grande variabilidade interanual de cerca de $40 \%$ (REDDY, 1986, apud BRITO et al., 2006)

A área de estudo apresenta quatro principais infraestruturas hidráulicas. A barragem de Massingir, o maior empreendimento hidráulico e o único reservatório de regularização de vazóes, que localiza-se no rio Elefantes. Esta barragem, projectada para que a sua represa armazenasse $2840 \mathrm{Mm}^{3}$ à cota de $125 \mathrm{~m}$, com uma superfície inundada de $151 \mathrm{~km}^{2}$, actualmente a sua capacidade está limitada a um volume de armazenamento de $1460 \mathrm{Mm}^{3}$. O açude de Macarretane, localizado ao longo do rio Limpopo, cujo objectivo

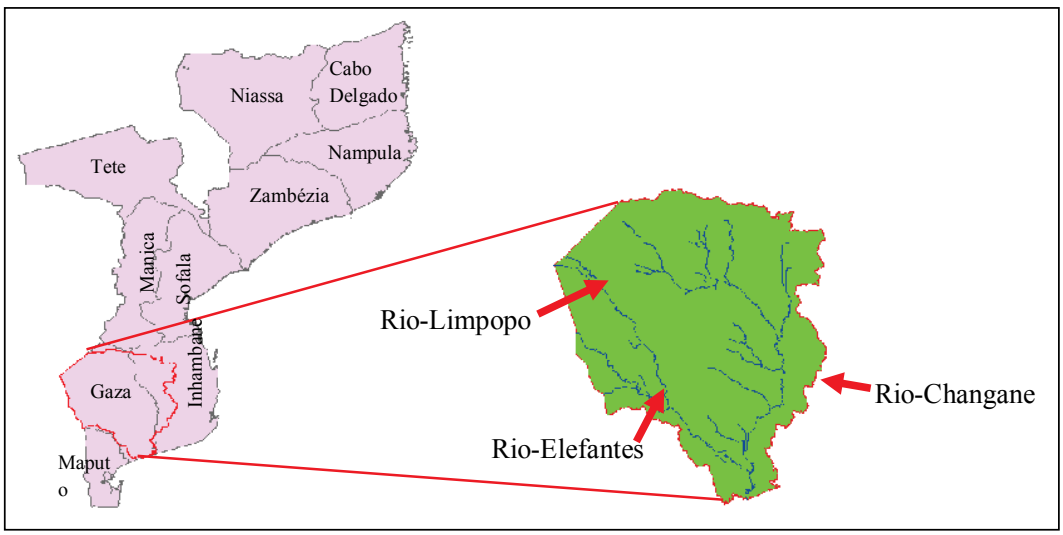

FIGURA 4. Rios das principais sub-bacias na parte moçambicana da bacia do Limpopo. Fonte: (Elaborado pelo autor; 2015). 
de sua construção é de assegurar o abastecimento de água ao perímetro irrigado de Chókwè, através de elevaçáo do nível das águas para a tomada do canal geral do regadio. E os regadios de Chókwè e do Baixo Limpopo, com uma área agricultável de cerca de 33 mil ha e $70 \mathrm{mil} \mathrm{ha,} \mathrm{respectivamente} \mathrm{(DNA,} \mathrm{1996).}$

Em termos de disponibilidades hídricas, pese embora Moçambique possuir uma rede hidrográfica considerável (Gomes et al., 1998), a região de estudo é dependente dos países a montante, contando como principais fontes de água os rios Elefantes e Limpopo que fluem desde a África do Sul para dentro do território nacional, para além de alguns afluentes locais com regime efémero.

A agricultura irrigada apresenta a maior demanda de água (LBTPC, 2010) para os dois regadios, Chókwè e Baixo Limpopo, que tem os cultivos de arroz, milho, tomate e feijóes como suas principais culturas. Outras demandas que não são menos importantes são as necessidades hídricas urbanas (cidade de Chókwè e de Xai-Xai) e rurais dispersas na bacia, que na sua maioria exploram águas subterrâneas, e a vazáo mínima de $7.5 \mathrm{~m}^{3} / \mathrm{s}$ estabelecida como forma de evitar a intrusão salina (DNA, 1996).

\section{Dados e procedimentos metodológicos utilizados}

De forma resumida são apresentados a seguir os principais dados e metodologia utilizados neste trabalho. O desenvolvimento mais detalhado pode ser encontrado em LIVELE, Virgílio António (2015).

Para o prosseguimento desta pesquisa, diferentes informaçóes sobre o objecto de estudo foram solicitadas em distintas instituiçóes e/ou entidades, merecendo distaque as seguintes: Administração Regional de Águas do Sul (ARA-Sul); Unidade de Gestáo do Bacia do Limpopo (UGBL); Serviços Distritais das Atividades Económicas (SDAEs), Direcção
Provincial de Agricultura-Gaza (DPAG); Hidráulica de Chókwè-Empresa Pública (HICEP) e Regadio do Baixo Limpopo (RBL). Foram colectados dados diários das séries históricas fluviométricas de vários postos dentro da área estudada, tendo sido utilizados dados das estaçóes localizadas nos rios Elefantes e Limpopo (figura 5). As estaçóes do rio Limpopo, E0033 e E0037, com períodos compreendidos entre $28 / 02 / 61$ à $31 / 08 / 13$ e de 20/01/71 à 29/02/12, respectivamente. E o posto do rio Elefantes, E0039, com uma série de 01/10/2003-31/12/13.

Porque as séries das três estaçóes apresentavam falhas, e havendo necessidade de homogeneização dos seus períodos, foi aplicado o método de regressão linear em Microsoft Excel ${ }^{\circ}$ para o preenchimento das lacunas e/ou complementaçáo dos dados, tendo se escolhido como intervalo das séries os anos de 1971 à 2013, com um total de 43 anos de dados.

Outros dados necessários foram as demandas hídricas dos principais usuários. Para tal, foi considerada a vazão mínima de $7,5 \mathrm{~m}^{3} / \mathrm{s}$, e estimou-se as necessidades hídricas mensais actuais (2013) e futuras (2017) para o abastecimento doméstico e de forma agrupada aferiu-se também as demandas dos usuários agrícolas/económicos, tabela 2. As demandas de água para as Associaçóes Agrícolas e os regadios de Chókwè e do Baixo Limpopo são para a satisfação hídrica das suas principais culturas praticadas, que são: arroz, milho, tomate e feijóes. E às empresas privadas as suas demandas são para as culturas da banana orgância e da cana-sacarina.

Para a análise que se pretendia nesta pesquisa e a metodologia utilizada, precisou-se também conhecer as características físicas da barragem de Massingir: volume inicial, mínimo e máximo; suas taxas de evapotranspiraçáo mensal com precipitação já descontada, e a curva cota-área-volume. Vale realçar que no volume máximo da barragem, tomou-se em afeição dois valores da sua capacidade, $1463 \mathrm{Mm}^{3}$,

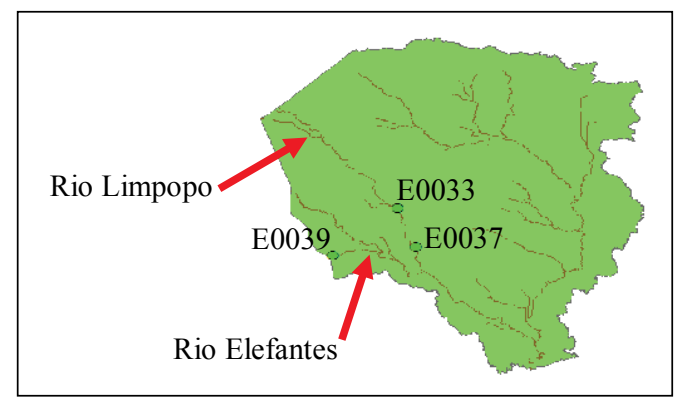

FIGURA 5. Postos das séries históricas fluviométricas utilizados neste trabalho.

Fonte: (Elaborado pelo autor; 2015). 
correspondente a capacidade actual de operação do reservatório na cota $115 \mathrm{~m}$, e o segundo de $2836 \mathrm{Mm}^{3}$, capacidade máxima do reservatório quando operado no seu nível pleno de armazenamento (NPA), na cota $125 \mathrm{~m}$ (tabela 3). $\mathrm{O}$ açude de Macarretane uma vez que serve apenas de um nó/represa de elevação do nível das águas para o seu desvio à tomada do canal geral do regadio do Chókwè, e não como reservatório de armazenamento, foi assumido como um "ponto de passagem".

TABELA 2

Demandas hídricas médias mensais actuais e futuras referentes aos principais usuários na parte moçambicana da bacia do Limpopo.

\begin{tabular}{|c|c|c|c|c|c|c|c|c|c|c|c|c|c|}
\hline \multicolumn{14}{|c|}{ Situação Actual (2013) } \\
\hline \multicolumn{14}{|c|}{ Demandas hídricas médias mensais dos principais usários na parte moçambicana da BRL } \\
\hline \multirow{2}{*}{\multicolumn{2}{|c|}{ Usuários }} & \multicolumn{4}{|c|}{ Económicos } & \multicolumn{5}{|c|}{ Não económicos } & & & \\
\hline & & Reg_Chokwe & \multicolumn{2}{|c|}{ Reg_BLimp } & Ass_Agricola & \multicolumn{2}{|c|}{ Abast_Domest } & \multicolumn{3}{|c|}{ Qmin } & & & \\
\hline \multicolumn{2}{|c|}{ Demandas (m3/s) } & 8,570 & \multicolumn{2}{|l|}{7,910} & 5,270 & \multicolumn{2}{|c|}{2,403} & \multicolumn{3}{|c|}{7,500} & & & \\
\hline \multicolumn{14}{|c|}{ Situação Futura (2017) } \\
\hline \multicolumn{14}{|c|}{ Demandas hídricas médias mensais $\mathrm{d}$} \\
\hline \multirow{2}{*}{\multicolumn{2}{|c|}{ Usuários* }} & \multicolumn{9}{|c|}{ Económicos } & \multicolumn{3}{|c|}{ Não económicos } \\
\hline & & Reg_Chokwe & \multicolumn{2}{|c|}{ Reg_BLimp } & Ass_Agricola & \multicolumn{2}{|c|}{ TAFC } & Empre & esas_C & ana & Abas & t_Domest & Qmin \\
\hline Demandas & $(\mathrm{m} 3 / \mathrm{s})$ & 15,720 & 27,680 & & 5,270 & &, 270 & & 8,990 & & & 2,560 & 7,500 \\
\hline $\begin{array}{l}{ }^{*} \text { Reg_Chokv } \\
\text { Domest.- Ab } \\
\text { gância) e Em }\end{array}$ & $\begin{array}{l}\text { ve- Regad } \\
\text { astecimen } \\
\text { presas.C }\end{array}$ & $\begin{array}{l}\text { dio de Chókw } \\
\text { nto Domésticc } \\
\text { Jana: agrupam }\end{array}$ & $\begin{array}{l}\text { è; Reg_BL } \\
\text { o; Qmin- Va } \\
\text { hento das er }\end{array}$ & $\begin{array}{l}\text { imp- Re } \\
\text { azão mír } \\
\text { mpresas }\end{array}$ & $\begin{array}{l}\text { egadio do } \\
\text { nima; TAF } \\
\text { s privadas }\end{array}$ & $\begin{array}{l}\text { Baixo Li } \\
=\text { C- Empr } \\
\text { de prodı }\end{array}$ & $\begin{array}{l}\text { impopo; } \\
\text { resa pri } \\
\text { ução de }\end{array}$ & $\begin{array}{l}\text { Ass.Ag } \\
\text { vada: } \mathrm{Th} \\
\text { cana-sa }\end{array}$ & $\begin{array}{l}\text { jicola- } \\
\text { le Africe } \\
\text { carina ( }\end{array}$ & & $\begin{array}{l}\text { ociad } \\
\text { Eood } \\
\text { ri-Su }\end{array}$ & $\begin{array}{l}\text { s Agrícol } \\
\text { ompany (b } \\
\text { da; CAM }\end{array}$ & $\begin{array}{l}\text { las; Abast. } \\
\text { banana or- } \\
\text { e MAI,SA). }\end{array}$ \\
\hline Dad & los de en & trada no Mo & delo Acqua & anet nar & $\begin{array}{l}\text { TABEL } \\
\text { Rese }\end{array}$ & A 3 & de Mas & ssinair & "Acu & & Ma & rretane' & \\
\hline Dao & los de en & trada no Mo & delo Acquá & anet par & ra o Rese & rvatorio & o de Mas & ssingir $\epsilon$ & "AçU & e & de Ma & rretane & 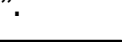 \\
\hline & & & aracterística & as físicas & da barrag & gem de $M$ & Iassingir & re Açude & de Ma & car & retane & & \\
\hline & & & & Volum & ies Caract & erísticos & (Mm3) & & & & & & \\
\hline Reservatório & & Volun & me Inicial & & Volume & Mínimo & & Volur & ne Máxi & & & olume met & ta $(100 \%)$ \\
\hline & & Opera. Act & NPA & Oper & ra. Actual & $\mathrm{NP}$ & $\mathrm{A}$ & Opera. A & ctual & $\mathrm{NP}$ & & & \\
\hline Barragem de & Massingi & 1323 & 2696 & & 140 & 140 & 10 & 1323 & & 269 & & 1,0 & \\
\hline Açude de Ma & carretane & 0,0 & 0,0 & & 0,0 & 0,0 & 0 & 4,0 & & 4, & & 0,0 & \\
\hline & & & urva Cota- $A$ & Área-Vol & lume da Ba & arragem & de Mass & singir & & & & & \\
\hline & & & Cota $(\mathrm{n}$ & & $\mathrm{ea}\left(\mathrm{km}^{2}\right)$ & Volume & $\left(\mathrm{Mm}^{3}\right)$ & & & & & & \\
\hline & & & 98,39 & & 30 & 140 & 10 & & & & & & \\
\hline & & & 102,36 & & 52 & 302 & 2 & & & & & & \\
\hline & & & 106,45 & & 82 & 576 & 6 & & & & & & \\
\hline & & & 110,28 & & 103 & 932 & 2 & & & & & & \\
\hline & & & 113,34 & & 116 & 126 & 66 & & & & & & \\
\hline & & & 115 & & 122 & 146 & 63 & & & & & & \\
\hline & & & 116,2 & & 126 & 161 & 17 & & & & & & \\
\hline & & & 118,35 & & 133 & 189 & 90 & & & & & & \\
\hline & & & 120,14 & & 138 & 213 & 32 & & & & & & \\
\hline & & & 122,56 & & 145 & 247 & 75 & & & & & & \\
\hline & & & 125 & & 151 & 283 & 36 & & & & & & \\
\hline & & & & Taxa d & le Evapor & ação (m & n/mês) & & & & & & \\
\hline Meses & Outubro & Novembro & Dezembro & Janeiro & Feveriro & Março & Abril & Maio & Junho & & Julho & Agosto & Setembro \\
\hline Evaporação & 0,2118 & 0,1972 & 0,1958 & 0,2108 & 0,2027 & 0,1834 & 0,1390 & 0,1278 & 0,1136 & & 1250 & 0,1498 & 0,1913 \\
\hline
\end{tabular}

Fonte : (DNA, 1996; ARA-SUL, 2014). 
É importante frisar que, actualmente o sistema (barragem de massingir) é operado de uma forma simples, em que independentemente da quantidade de água disponível no reservatório, e que estação do ano, sempre que tiver água suficiente (acima do volume mínimo), o volume liberado do reservatório procura sempre igualar as quantidades demandadas para a irrigação agrícola, principal usuário, e outros usos.

\section{Determinação das curvas de demanda de água para os principais usuários económicos}

As funçóes de demanda de água para os usuários agrícolas foram determinadas através do método Point Expansion, acima descrito. Para a aplicação deste método, de forma sucinta obedeceu-se os seguintes procedimentos: (i) cômputo da quantidade total anual de água (w) necessária para cada usuário agrícola, que foi a multiplicação da área total pela quantidade total de água demandada para todas culturas por hectare, (ii) cálculo do custo total de água (preço-p) pago pelos agricultores para ter a água disponível nos seus campos; através da soma da tarifa paga em cada $\mathrm{m}^{3}$ e do custo de bombeamento de água, e (iii) determinação das funçóes de demanda, usando a forma potencial. Para além do ponto de cada usuário (w,p), foi necessário conhecer a elasticidade preço da demanda. Com isto,, devido a inexistência de dados na área de estudo para estimar este valor, achou-se com validade científica considerar um valor que representasse o comportamento dos agricultores da regiáo da bacia do Limpopo, tendo se utilizado $\varepsilon=-0,19$, valor estimado por Walter et al. (2011), na sub-bacia do rio Elefantes. Na tabela 4, encontram-se resumidos os resultados dos pontos das curvas de demanda pelo uso da água por cada usuário.

E na tabela 5 são apresentadas as equaçóes ou funçóes de demanda dos usuários que possibilita-

TABELA 4

Pontos das curvas de demanda pelo uso da água por cada usuário

\begin{tabular}{|c|c|c|c|c|c|c|c|}
\hline \multirow[t]{2}{*}{ Usuário } & \multirow{2}{*}{$\begin{array}{c}\begin{array}{c}\text { Área total } \\
\text { considerada } \\
\text { (ha) }\end{array} \\
\text { (a) }\end{array}$} & \multirow{2}{*}{$\begin{array}{c}\text { Consumo } \\
\text { médio anual } \\
\text { por hectar } \\
\text { (m3) } \\
\text { (b) }\end{array}$} & \multirow{2}{*}{$\begin{array}{c}\text { Consumo } \\
\text { médio } \\
\text { anual }(W) \\
\mathrm{m} 3 *^{*}\left(10^{\wedge} 3\right) \\
(\mathrm{c})=\mathrm{a}^{*} \mathrm{~b} \\
\end{array}$} & \multirow{2}{*}{$\begin{array}{c}\text { *Tarifa de } \\
\text { água }(\mathrm{Mt} / \mathrm{m} 3) \\
\text { (d) }\end{array}$} & \multirow{2}{*}{$\begin{array}{c}* * \text { Custo de } \\
\text { bombeamento } \\
(\mathrm{Mt} / \mathrm{m} 3)\end{array}$} & \multicolumn{2}{|c|}{$\begin{array}{l}\text { Custo total de água (P) } \\
\qquad(\mathrm{f})=(\mathrm{d}+\mathrm{e}) * 1000\end{array}$} \\
\hline & & & & & & Mt/1000 m3 & US\$ $/ 1000 \mathrm{~m} 3$ \\
\hline Regadio de Chókwè & 13000 & 20500 & 266500 & 0,111 (i) & 0,763 & 874 & 27,08 \\
\hline $\begin{array}{l}\text { Regadio do Baixo } \\
\text { Limpopo }\end{array}$ & 12000 & 20500 & 246000 & 0,096 (ii) & 0,763 & 859 & 26,61 \\
\hline Associações Agrícolas & 8000 & 20500 & 164000 & 0,096 (iii) & 0,763 & 859 & 26,61 \\
\hline TAFC, Ltd & 500 & 17000 & 8500 & 0,08 (iv) & 0,763 & 843 & 26,12 \\
\hline $\begin{array}{l}\text { Empresas de Cana- } \\
\text { sacarina }\end{array}$ & 63000 & 19250 & 1212750 & $0,096(\mathrm{v})$ & 0,763 & 859 & 26,61 \\
\hline
\end{tabular}

TABELA 5

Funções de demanda da água de cada usuário e equações invertidas para $P$.

\begin{tabular}{c|l|l|l}
\hline Nr. & \multicolumn{1}{|c|}{ Usuário } & $\begin{array}{c}\text { Funções de demanda da } \\
\text { água }\end{array}$ & $\begin{array}{c}\text { Funções da demanda de água } \\
\text { invertidas para } \mathbf{P}\end{array}$ \\
\hline 1 & Regadio de Chókwè & $\mathrm{W}=965082,859^{*} \mathrm{P}^{\wedge}-0,19$ & $\mathrm{P}=\left((1 / 965082,859)^{*} \mathrm{~W}\right)^{\wedge}-(1 / 0,19)$ \\
\hline 2 & Regadio do Baixo Limpopo & $\mathrm{W}=888004,304^{*} \mathrm{P}^{\wedge}-0,19$ & $\mathrm{P}=\left((1 / 888004,304)^{*} \mathrm{~W}\right)^{\wedge}-(1 / 0,19)$ \\
\hline 3 & Associações Agrícolas & $\mathrm{W}=592002,869^{*} \mathrm{P}^{\wedge}-0,19$ & $\mathrm{P}=\left((1 / 592002,869)^{*} \mathrm{~W}\right)^{\wedge}-(1 / 0,19)$ \\
\hline 4 & TAFC, Ltd & $\mathrm{W}=30573,6881^{*} \mathrm{P}^{\wedge}-0,19$ & $\mathrm{P}=\left((1 / 30573,6881)^{*} \mathrm{~W}\right)^{\wedge}-(1 / 0,19)$ \\
\hline 5 & Empresas de Cana-sacarina & $\mathrm{W}=4377752,92^{*} \mathrm{P}^{\wedge}-0,19$ & $\mathrm{P}=\left((1 / 4377752,92)^{*} \mathrm{~W}\right)^{\wedge}-(1 / 0,19)$ \\
\hline
\end{tabular}


ram a expansão/construção do resto das curvas, e as funçóes invertidas para $\mathrm{p}$, como forma de permitir a determinação dos benefícios totais obtidos pelos usuários no consumo da água.

A partir destas funçôes de demanda da água foram plotadas as curvas de demanda ilustradas na figura 6, que representam a disposição que os usuários têm

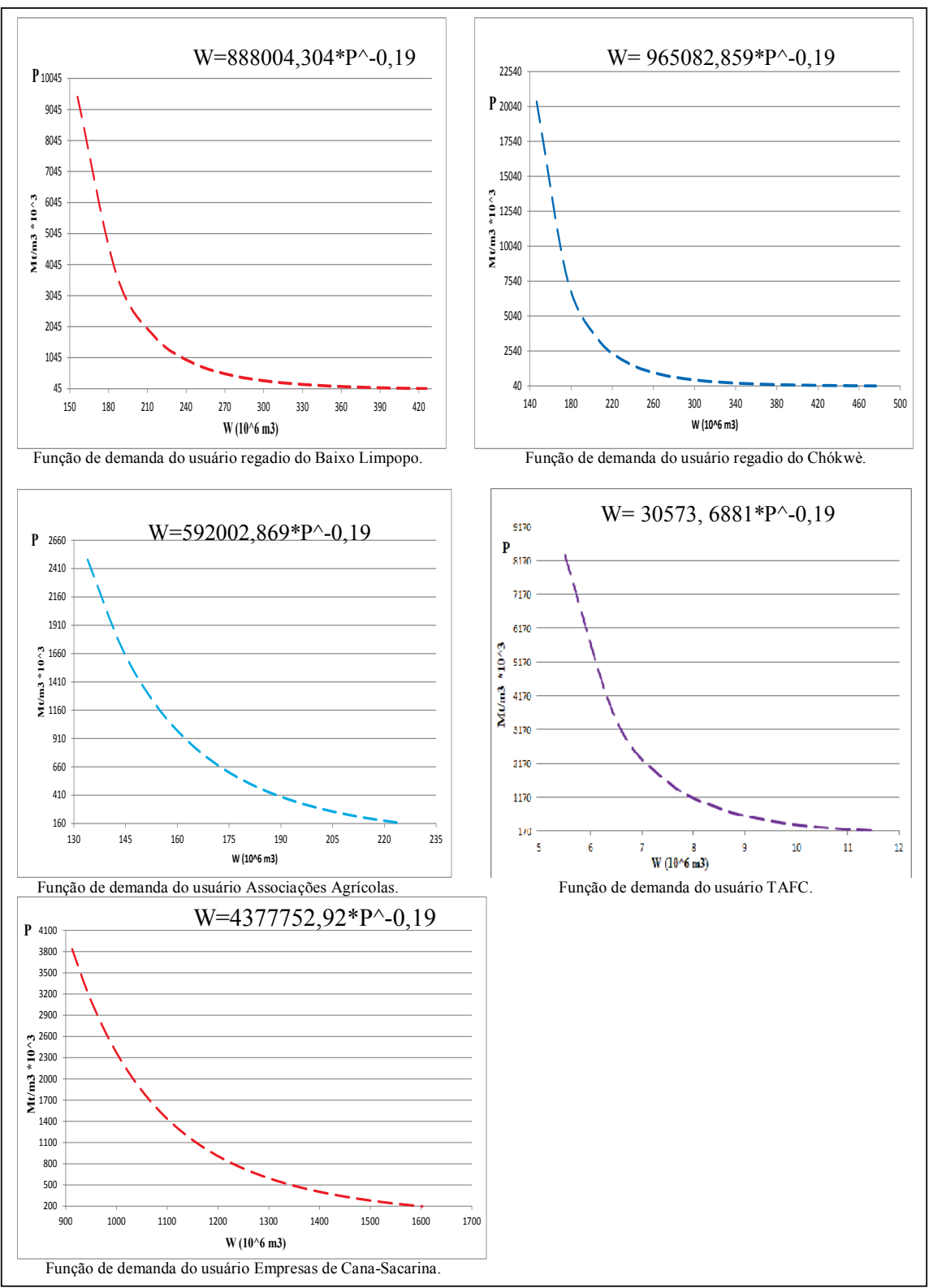

FIGURA 6.

Funções de demanda da água dos principais usuários na parte moçambicana da bacia do Limpopo. 
as curvas mostram que os agricultores estariam menos dispostos a pagar preços muito altos, o que obrigaria a redução do consumo de água.

\section{Utilização do modelo Acquanet para avaliação dos impactos económicos}

A avaliação dos impactos económicos da água alocada ou que pode ser fornecida entre usuários agrícolas, e ou escassez na operação do sistema hídrico da parte moçambique da bacia, foi possível através de definição e simulação de cenários no Acquanet, no seu submodelo de Análise Económica, tendo sido feita uma simulação Contínua com período total de 43 anos (516 meses), intervalo ou variaçáo do tempo mensal, e opção de cálculo Calibração. Para a aplicação deste submodelo teve-se o seguinte processo: (i) representação do sistema hídrico estudado em rede de fluxo; (ii) entrada dos dados segundo os cenários definidos; (iii) criaçáo das curvas de benefícios marginais e (iv) determinação automática das capacidades máximas e benefícios dos links económicos.

Quanto ao primeiro processo, foram representadas duas topologias do sistema analisado segundo as demandas actuais e futuras, acima apresentadas na tabela 2. Uma topologia considerou apenas a situação actual (2013), tanto demandas económicas como não económicas, incluindo as necessidades da vazão mínima. E a segunda representação, para além de todas demandas actuais, incluiu a demandas futuras projectadas para o ano 2017. De seguida, foram definidos quatro principais cenários dentro das regras actuais de operação da barragem de Massingir:

Cenário A (Cen.A): este cenário foi testado com principal objectivo de conhecer e avaliar o nível de satisfação de todas demandas actuais
(2013) e seus impactos económicos acumulados pelos agricultores, dentro da capacidade operacional actual da barragem Massingir $\left(1463 \mathrm{Mm}^{3}\right)$;

Situaçáo B (Cen.B): pretendendo avaliar a capacidade do sistema hídrico estudado quanto a sua capacidade no atendimento de mais demandas, para além das actuais, simulou-se a situação $\mathrm{B}$, tendo se considerado a soma de todas as necessidades hídricas actuais e futuras estimadas para 2017, e assumindo o mesmo volume actual de armazenamento da barragem de Massingir $\left(1463 \mathrm{Mm}^{3}\right)$;

Terceiro cenário (Cen.C): este caso, considerou o mesmo objectivo do cenário $\mathrm{B}$, se diferenciando apenas no volume de armazenamento da barragem de Massingir, pois, aqui se assumiu a capacidade máxima do reservatório $\left(2836 \mathrm{Mm}^{3}\right)$, em suas condiçóes de NPA; e

Último caso (Cen.D): nesta situação considerou-se também a capacidade máxima do reservatório de Massingir, porém, as séries fluviómetricas, dados de entrada para as vazóes naturais/afluentes na barragem de Massingir (rio Elefantes) e no rio Limpopo, foram estimadas considerando as projecçôes de consumo de água nos países a montante (África do Sul, Zimbabwé e Botswana).

E por último, através as funçôes de ajuste exponencial (tabela 6) obtidas nas curvas de demanda de água acima determinadas (tabela 5), foram criadas as curvas de benefícios marginais para cada usuário agrícola no sistema, e automaticamente o modelo computou as capacidades máximas e benefícios dos links económicos.

TABELA 6

Funções ajustadas das curvas de demanda da água para a determinação do benefício marginal obtido por cada usuário económico na parte moçambicana da BRL

\begin{tabular}{c|l|l|c}
\hline Nr. & \multicolumn{1}{|c|}{ Usuário } & \multicolumn{1}{|c|}{$\begin{array}{c}\text { Funções de benefício } \\
\text { Marginal Ajustadas }\end{array}$} & $\begin{array}{c}\text { coeficiente de } \\
\text { determinação } \\
\left(\mathbf{R}^{2}\right)\end{array}$ \\
\hline 1 & Regadio de Chókwè & $\mathrm{y}=\mathrm{Bmg}=154009^{*} \mathrm{e}^{\wedge}-0,018 * \mathrm{Q}$ & $\mathrm{R}^{2}=0,974$ \\
\hline 2 & Regadio do Baixo Limpopo & $\mathrm{y}=\mathrm{Bmg}=148616^{*} \mathrm{e}^{\wedge}-0,02 * \mathrm{Q}$ & $\mathrm{R}^{2}=0,9829$ \\
\hline 3 & $\begin{array}{l}\text { Associações Agrícolas e } \\
\text { outros }\end{array}$ & $\mathrm{y}=\mathrm{Bmg}=121885^{*} \mathrm{e}^{\wedge}-0,03 * \mathrm{Q}$ & $\mathrm{R}^{2}=0,9948$ \\
\hline 4 & TAFC, Ltd & $\mathrm{y}=\mathrm{Bmg}=227795^{*} \mathrm{e}^{\wedge}-0,641 * \mathrm{Q}$ & $\mathrm{R}^{2}=0,9888$ \\
\hline 5 & Empresas de Cana-sacarina & $\mathrm{y}=\mathrm{Bmg}=161139^{*} \mathrm{e}^{\wedge}-0,004 * \mathrm{Q}$ & $\mathrm{R}^{2}=0,9943$ \\
\hline
\end{tabular}




\section{RESULTADOS E DISCUSSÃO}

Antes de apresentar os resultados alcançados neste trabalho, há que lembrar que no sistema hídrico simulado e na sua representação topológica foram incluidas as demandas não económicas (abastecimento doméstico e a vazão mínima de controle da intrusão salina), entretanto, para o objectivo principal que se pretende com esta pesquisa, que é o de avaliar o impacto económico da satisfação e/ou escassez hídrica entre os agricultores, são apenas ilustrados a seguir os resultados dos usuários económicos.

\section{Benefícios económicos médios anuais alcançados pelos usuários agrícolas nos cenários simulados}

De forma geral, a tabela 7 mostra os benefícios totais agrícolas alcançados pelos principais usuários, em função do nível de satisfação das suas demandas hídricas mensais nos quatro diferentes cenários a posterior propostos e simulados. No cenário A (simulação do sistema apenas com as demandas actuais (2013)) os resultados mostram que actualmente o sistema consegue dar uma garantia de provimento de água a $100 \%$, sem registo de falhas, o que possibilita aos usuários económicos obter ao máximo os benefícios anuais advindos do uso da água, sem nenhuma perda económica, que ocorreria caso houvesse disponibilidade restrita ou escassez de água.

No cenário B, que considerou-se as demandas actuais e futuras estimadas para 2017, houve uma realocaçáo de água segundo as novas vazóes necessárias entre os usuários e suas prioridades de atendimento. Com isto, contrariamente ao cenário anterior, os resultados obtidos nesta simulação apresentam dé- ficits na satisfação de todas demandas consideradas na análise, mostrando que dentro das regras actuais de operacionalização do sistema hídrico estudado, limitaçóes físicas da barragem de Massingir e fluxos não regularizados no rio Limpopo, o sistema é incapaz de dar uma garantia de atendimento a $100 \%$ às totais futuras demandas de água projectadas para 2017 na parte moçambicana da bacia do Limpopo. E por conta deste atendimento abaixo dos volumes demandados, os agricultores viram os seus possíveis benefícios máximos anuais reduzidos em uma média aritmética de um pouco mais de $6 \%$, representando o seguinte para cada usuário: Ass_Agricola uma diminuição de $9,5 \%\left(677.091 \times 10^{\wedge} 3 \mathrm{Mt}\right)$ devido a redução na satisfação das suas necessidades mensais, de 5,27 $\mathrm{m}^{3} / \mathrm{s}$ para $4,77 \mathrm{~m}^{3} / \mathrm{s} ;$ Empresas_Cana com $4,57 \%$ dos ganhos económicos não alcançados; Reg_Blimp: 9\% de redução nos seus possíveis benefícios máximos em condiçốes de satisfação total das suas necessidades hídricas; Reg_Chokwe 7,4\%, e a TAFC com cerca de $1,55 \%\left(10.508,7 \times 10^{\wedge} 3 \mathrm{Mt}\right)$ de diminuição dos seus ganhos. Vale realçar que os resultados dos usuários Reg_BLimp e Reg_Chokwe obtidos nos cenários B, $\mathrm{C}$ e D, estão sujeitos a limitação do método Point Expansion (pouco confiáveis), pelo facto de a quantidade média anual de água fornecida a cada um estar muito afastada do ponto real utilizado na expansão das suas funçóes de demanda.

Ao se mudar do cenário B para o $\mathrm{C}$ (política de operaçáo actual, entretanto assumindo a capacidade máxima "NPA"), percebe-se que os usuários agrícolas registaram um incremento considerável nos seus benefícios médios anuais, chegando aproximadamente uma média 2,75\% de aumento nos ganhos. Na sequência, os usuários conseguiram os seguintes incrementos:

TABELA 7

Benefícios económicos ganhos pelos usuários agrícolas no atendimento das suas demandas hídricas

\begin{tabular}{|c|c|c|c|c|c|c|c|c|c|c|c|c|c|}
\hline \multirow[b]{2}{*}{ Demandas } & \multirow[b]{2}{*}{$\begin{array}{l}\text { Usuários de } \\
\text { demandas } \\
\text { económicas }\end{array}$} & \multicolumn{4}{|c|}{ Cenário $\mathrm{A}$} & \multirow[b]{2}{*}{$\begin{array}{c}\text { *Qmed. } \\
\text { nec. } \\
\left(\mathrm{m}^{3} / \mathrm{s} / \mathrm{mês}\right)\end{array}$} & \multicolumn{2}{|c|}{ Cenário $B$} & \multicolumn{2}{|c|}{ Cenário $\mathrm{C}$} & \multicolumn{2}{|c|}{ Cenário D } & \multirow{2}{*}{$\begin{array}{c}* * \text { benefício } \\
\text { máximo anual } \\
\text { possível nos } \\
\text { cenários } \mathrm{B}, \mathrm{C} \text { e D } \\
\left(10^{3} \mathrm{Mt} / \text { ano }\right)\end{array}$} \\
\hline & & $\begin{array}{c}\text { Qmed. } \\
\text { nec. } \\
\left(\mathrm{m}^{3} / \mathrm{s} / \mathrm{mês}\right)\end{array}$ & $\begin{array}{c}\text { Qmed. } \\
\text { fornec. } \\
\left(\mathrm{m}^{3} / \mathrm{s} / \mathrm{mês}\right)\end{array}$ & $\begin{array}{l}\text { benefício } \\
\text { médio anual } \\
\left(10^{3} \mathrm{Mt} / \mathrm{ano}\right)\end{array}$ & $\begin{array}{c}\text { benefício } \\
\text { máximo anual } \\
\text { possível } \\
\left(10^{3} \mathrm{Mt} / \mathrm{ano}\right)\end{array}$ & & $\begin{array}{c}\text { Qmed. } \\
\text { fornec. } \\
\left(\mathrm{m}^{3} / \mathrm{s} / \mathrm{mês}\right)\end{array}$ & $\begin{array}{c}\text { benefício } \\
\text { médio anual } \\
\left(10^{3} \mathrm{Mt} / \mathrm{ano}\right)\end{array}$ & $\begin{array}{c}\text { Qmed. } \\
\text { fornec. } \\
\left(\mathrm{m}^{3} / \mathrm{s} / \mathrm{mês}\right)\end{array}$ & $\begin{array}{c}\text { beneficio } \\
\text { médio anual } \\
\left(10^{3} \mathrm{Mt} / \mathrm{ano}\right)\end{array}$ & $\begin{array}{c}\text { Qmed. } \\
\text { fornec. } \\
\left(\mathrm{m}^{3} / \mathrm{s} / \mathrm{mês}\right)\end{array}$ & $\begin{array}{c}\text { beneficio } \\
\text { médio anual } \\
\left(10^{3} \mathrm{Mt} / \mathrm{ano}\right)\end{array}$ & \\
\hline \multirow{5}{*}{ Económicas } & Reg_BLimp & 7,91 & 7,91 & $13.046 .940,0$ & $13.046 .940,0$ & 27,68 & 25,155 & $34.521 .510,0$ & 26,162 & $35.897 .190,0$ & 25,118 & $34.451 .870,0$ & $37.946 .840,0$ \\
\hline & Reg_Chokwe & 8,57 & 8,57 & $14.678 .310,0$ & $14.678 .310,0$ & 15,72 & 14,535 & $23.442 .710,0$ & 15,041 & $24.241 .450,0$ & 14,38 & $23.168 .660,0$ & $25.307 .710,0$ \\
\hline & Ass_Agricola & 5,27 & 5,27 & $7.130 .166,0$ & \begin{tabular}{|l|}
$7.130 .166,0$ \\
\end{tabular} & 5,270 & 4,77 & $6.453 .075,0$ & 4,964 & $6.715 .620,0$ & 4,774 & $6.458 .871,0$ & $7.130 .166,0$ \\
\hline & TAFC & & & & & 0,270 & 0,266 & $667.308,4$ & 0,267 & $671.249,4$ & 0,266 & $667.308,6$ & $677.817,1$ \\
\hline & Empresas_Cana & & & & & 38,990 & 37,162 & $66.619 .670,0$ & 37,995 & $68.077 .440,0$ & 36,65 & $65.706 .630,0$ & $69.812 .890,0$ \\
\hline
\end{tabular}

*vazões médias mensais necessárias para cada usuário, utilizadas na simulação dos últimos três cenários (B,C e D).

${ }^{*}$ nos últimos três cenários $(B, C$ e $D)$, devido a não variação da quantidade mensal de água necessária para cada usuário em todos os cenários, os possíveis benefícios máximos anuais mantiveram-se constantes. 
Ass_agricola aumentou $262.545^{*} 10^{\wedge} 3 \mathrm{Mt} / \mathrm{ano}$ Reg_Blimp 1.375.680*10^3 Mt/ano; Reg_Chokwe com 798.740*10^3 Mt/ano; TAFC 3.941*10^3 Mt/ano e Empresas_Cana $1.457 .770^{*} 10^{\wedge} 3 \mathrm{Mt} / \mathrm{ano}$. E comparando os casos $\mathrm{C}$ e D (política de operaçáo actual, assumindo a capacidade máxima "NPA" e considerando as projecçóes de consumo dos países de montante) vê-se que o incremento de ganhos relacionado com o cenário C (2,75\%), aqui neste caso $\mathrm{D}$, diminuiu em uma média aritmética de cerca de 0,55\%, mostrando o grau de impacto negativo que terá-se com o aumento de abstracçôes de água pelos países de montante.

\section{Custos económicos acumulados pelos usuários devido a escassez/déficit de água}

A tabela 8 apresenta de forma sumarizada a distribuição global das perdas económicas acumuladas pelos usuários agrícolas na área de pesquisa. Dos quatro cenários simulados neste estudo, os resultados aqui mostrados correspondem apenas a três casos, B, $\mathrm{C}$ e $\mathrm{D}$, pelo facto de no cenário $\mathrm{A}$ os usuários não terem registado perdas económicas.

Devido o não atendimento a $100 \%$ das vazôes médias mensais demandadas pelos usuários, estes, foram acumulando a cada mês custos económicos. Dentro das condiçóes de simulação dos três cenários mostrados (tabela 8), na situação $\mathrm{B}$ os usuários registaram em média uma perda anual de 1.834.229,9*10^3 Mt/ano, na qual o Reg_Blimp aparece com maior perda, $3.425 .330^{*} 10^{\wedge} 3 \mathrm{Mt} / \mathrm{ano}$, correspondente ao acúmulo de déficit mensal de $2,525 \mathrm{~m}^{3} / \mathrm{s}$ durante todo o período simulado. Ao se confrontar os resultados, vê-se que deste cenário (B) para o $\mathrm{C}$, os usuários tiveram seus custos reduzidos em um pouco mais de $40 \%$, isto porque houve diminuição de escassez de água em uma média de aproximadamente igual a 38,4\%. Entretanto, no caso D, cenário que apresentou os maiores custos econômicos, os resultados mostram uma situação inversa, na qual as perdas econômicas acumuladas no caso $\mathrm{C}$ aumentaram para todos os usuários agrícolas, com um incremento médio aproximado de $86 \%$, equivalentes a uma subida de custos médios anuais de 1.029.922,0*10^3 Mt/ano. Para os custos econômicos registados neste cenário (D) como consequência de aumento de escassez de água, merecem ainda destaque os usuários Reg_Chokwe e Empresas_Cana que obtiveram mais de $100 \%$ (1.072.790*10^3 Mt/ano e 2.370.810*10^3 Mt/ano respectivamente) de incremento de perdas quando relacionados com os resultados da situação C.

\section{CONCLUSÕES}

O modelo de análise económica aplicado neste trabalho mostrou que para obtenção dos benefícios máximos económicos de alocação de água, é preciso que a satisfação das necessidades hídricas seja a 100\%. $\mathrm{E}$ que a escassez de água e/ou atendimento abaixo dos volumes demandados pode levar a acúmulo de perdas económicas. Dos resultados alcançados pôde se verificar que, dentro das condiçôes actuais de operação do sistema hídrico da parte moçambicana da bacia do Limpopo, o sistema consegue garantir a obtenção de benefícios máximos apenas aos usuários agrícolas actuais. Entretanto, o mesmo sistema apresenta-se incapaz de poder acomodar as demandas futuras projectadas para 2017 e satisfazer na sua totalidade todas necessidades de água, o que poderá, consequentemente, no futuro gerar custo de escassez de água aos usuários.

Havendo disponibilidade hídrica, a operação da barragem de Massingir na sua capacidade máxima (nível pleno de armazenamento), mostrou-se neste estudo como uma solução alternativa que pode futuramente minimizar os déficits de água no atendimento das demandas de todos os principais usuários naquela área de estudo.

TABELA 8

Custos médios anuais de escassez de água para os usuários agrícolas, nos diferentes cenários simulados (B,C e D).

\begin{tabular}{|c|c|c|c|c|c|c|c|c|c|c|}
\hline \multirow[b]{2}{*}{$\begin{array}{l}\text { Us uários de } \\
\text { de mandas } \\
\text { económicas }\end{array}$} & \multirow[b]{2}{*}{$\begin{array}{c}\text { Qmed. } \\
\text { nec. } \\
\left(\mathrm{m}^{3} / \mathbf{s} / \mathbf{m e ̂ s}\right)\end{array}$} & \multicolumn{3}{|c|}{ Cenário B } & \multicolumn{3}{|c|}{ Cenário $\mathrm{C}$} & \multicolumn{3}{|c|}{ Cenário D } \\
\hline & & $\begin{array}{c}\text { Qmed. } \\
\text { fornec. } \\
\left(\mathbf{m}^{3} / \mathbf{s} / \mathbf{m e ̂ s}\right)\end{array}$ & $\begin{array}{c}\text { Qmed. } \\
\text { Escassez/déficit } \\
\left(\mathbf{m}^{3} / \mathbf{s} / \mathbf{m e ̂ s}\right)\end{array}$ & $\begin{array}{l}\text { Custo médio } \\
\text { anual da } \\
\text { escassez de } \\
\text { água } \\
\left(10^{3} \mathrm{Mt} / \text { ano }\right) \\
\end{array}$ & $\begin{array}{c}\text { Qmed. } \\
\text { fornec. } \\
\left(\mathbf{m}^{3} / \mathbf{s} / \mathbf{m e ̂ s}\right)\end{array}$ & $\begin{array}{c}\text { Qmed. } \\
\text { escassez/défici } \\
\mathbf{t}\left(\mathbf{m}^{3} / \mathbf{s} / \mathbf{m e ̂ s}\right)\end{array}$ & $\begin{array}{c}\text { Custo mé dio } \\
\text { anual da } \\
\text { escassez de } \\
\text { água } \\
\left(10^{3} \mathrm{Mt} / \mathrm{ano}\right)\end{array}$ & $\begin{array}{c}\text { Qmed. } \\
\text { fornec. } \\
\left(\mathbf{m}^{3} / \mathbf{s} / \mathbf{m e ̂ s}\right)\end{array}$ & $\begin{array}{c}\text { Qmed. } \\
\text { escassez/dé ficit } \\
\left(\mathbf{m}^{3} / \mathbf{s} / \mathbf{m e ̂ s}\right)\end{array}$ & $\begin{array}{l}\text { Custo médio } \\
\text { anual da } \\
\text { escassez de } \\
\text { água } \\
\left(10^{3} \mathrm{Mt} / \mathrm{ano}\right)\end{array}$ \\
\hline Reg_BLimp & 27,68 & 25,155 & 2,525 & $3.425 .330,0$ & 26,162 & 1,518 & $2.049 .650,0$ & 25,118 & 2,562 & $3.494 .970,0$ \\
\hline Reg_Chokwe & 15,72 & 14,535 & 1,185 & $1.865 .000,0$ & 15,041 & 0,679 & $1.066 .260,0$ & 14,38 & 1,340 & $2.139 .050,0$ \\
\hline Ass_Agricola & 5,270 & 4,77 & 0,500 & $677.091,0$ & 4,964 & 0,306 & $414.546,0$ & 4,774 & 0,496 & $671.295,0$ \\
\hline TAFC & 0,270 & 0,266 & 0,004 & $10.508,7$ & 0,267 & 0,003 & $6.567,7$ & 0,266 & 0,004 & $10.508,5$ \\
\hline Empresas_Cana & 38,990 & 37,162 & 1,828 & $3.193 .220,0$ & 37,995 & 0,995 & $1.735 .450,0$ & 36,65 & 2,340 & $4.106 .260,0$ \\
\hline
\end{tabular}




\section{Referências}

ARA-SUL. Estações e Dados da bacia do Limpopo. Administração Regional de Águas do Sul, Maputo, 2014. Base de dados.

California Water Plan: Integrated Water Management, vol.1, update 2009. Disponivel em: <http://www.waterplan.water.ca.gov/cwpu2009/>. Acesso em: Julho de 2014.

BOGAERT, Sarah et al. (2012). The role of water pricing and water allocation in agriculture in deliverering sustainable water use in Europe. European Commission, Project number 11589.

BRITO, Rui et al. (2006). Profile of the Limpopo Basin in Mozambique: a contribution to the Challenge Program on Water and Food Project 17-"Integrated Water Resource Management for Improved Rural Livelihoods: Managing risk, mitigating drought and improving water productivity in the water scarce Limpopo Basin", Universidade Eduardo Mondlane, CP 257, Maputo, Moçambique.

DNA. Monografía hidrográfica da bacia do rio Limpopo, Relatório nr. 16a/96. Texto. Ministério de Obras Públicas e Habitação, Direcção Nacional de Águas, Maputo, 1996.

FAO. Dia mundial da água: Agricultura utiliza 70\% da água mundial; "Água e segurança alimentar". Disponível em:< https://www.fao.org. br/DMApcqcn15mladFAO.asp>. Acesso em: Junho/2014.

GOMES, Fernanda et al. (1998). Research Project on Water Demand Management in Southern Africa. Mozambique Country Study-Case Study: Chókwè Irrigation Scheme, $1^{\text {st }}$ Draft, Maputo.

GRIFFIN, Ronald C. Water Resource Economics: The Analysis of Scarcity, Policies, and Projects. London, England, The MIT Press, 2006. LABADIE, John W. MODSIM 8.1: River Basin Management-Decision Support System-User Manual and Documentation. Department of Civil and Environmental Engineering, Colorado State University, 2010.

LABSID. Acquanet-Modelo para alocacação de água em sistemas complexos de recursos hídricos, Manual do Usuário. Escola Politécnica da Universidade de São Paulo. Departamento de Engenharia Hidráulica e Sanitária. Laboratório de Sistemas de Suporte a Decisões, 2002.

LBPTC. Limpopo Basin Permanent Technical Committee. Joint Limpopo River Basin Study Scoping Phase. Final Report/Main Report, Ministério de Obras Públicas e Habitação, Direcção Nacional de Águas, 2010.

Limpopo Basin Strategic Plan for Reducing Vulnerability to Floods and Droughts. Draft for Discussion with Riparian Governments. UN-HABITAT/UNEP. July 2007. pp 23.

MOLLE, François; BERKOFF, Jeremy. Irrigation Water Pricing: The Gap Between Theory and Practice, London, UK, 2007a.

PORTO, Rubem L.L. et al. (2003). Sistema de suporte a decisão para análise de sistemas de recursos hídricos.1-159p.

ROBERTO, Alexandre Nunes; PORTO,Rubem La Laina. Alocação da Água entre Múltiplos Usos em uma Bacia Hidrográfica: "Operação de Sistemas de Recursos Hídricos: modelos hidrológicos e ambientais, sistemas de suporte a decisão, Optimização de sistemas, sistemas inteligentes", 2001. Disponivel em: $<$ http://www.labsid.com.br/publicacoes.php?id=87\&tp=7>. Acesso em: Junho/2014.

ROBERTO, Alexandre Nunes. Modelos de Rede de Fluxo para Alocação da Água entre Múltiplos Usos em uma Bacia Hidrográfica. (2002). 116f. Dissertação de Mestrado em Engenharia Hidráulica e Sanitária, Escola Politécnica da Universidade de São Paulo, São Paulo, 2002.

ROBERTO, Alexandre Nunes et. al. (2007). 0 Novo Sistema de Suporte a Decisões acquanet. Disponível em:< http://www.labsid.com. br/publicacoes. php?id=87\&tp=7>. Acesso em: Junho/2014.

WALTER, Teresa; KLOOS, Julia; TSEGAI, Daniel. Options for improving water use efficiency under worsening scarcity: Evidence from the Middle Olifants Sub-Basin in South Africa. Water SA, South Africa, v. 37, no.3, July, 2011. ISSN 1816-7950

YOUNG, RobertA. Measuring Economics Benefits for Water Investments and Polices. World Bank Technical Paper no WTP. 338, The World Bank Washington, D. C.,1996.

Virgílio António Livele Mestrando em Recursos Hídricos e Saneamento Ambiental na Universidade Federal do Rio Grande do Sul-UFRGS, Instituto de Pesquisas Hidráulicas-IPH. Email:virgiliolivele@gmail.com.

André Luiz Lopes da Silveira Orientador do AUTOR na sua dissertaçáo de mestrado em Recursos Hídricos e Saneamento Ambiental na Universidade Federal do Rio Grande do Sul-UFRGS, Instituto de Pesquisas Hidráulicas-IPH. Email: andre@iph.ufrgs.br. 